\title{
A Simple and Cost Effective Data Acquisition System for Mobile Satellite Experimental Work under Tree- shadowed and Building-shadowed
}

\author{
V.P. Bong, W.A. Wan Zainal Abidin, I. Abba, A.K. Othman, H. Zen, T. Masri \\ Department of Electronic Engineering, \\ Universiti Malaysia Sarawak, 94300 Kota Samarahan, Sarawak, Malaysia.
}

\begin{abstract}
Signal degradation in Mobile Satellite (MS) is due to several factors such as ionospheric effect, multipath fading, treeshadowed and building-shadowed. This affects the Mobile Satellite Service (MSS) to provide continuous communication service to the users. Since there is not adequate data available on the signal performance of the MS in the Asian continent, thus, an experimental work is essential to be carried out to investigate the effect of factors such as the tree-shadowed and buildingshadowed on the signal strength. This paper discusses the cost effective set up. It can be used to measure the effect of trees and buildings on the received mobile satellite signal operating in the L-band. The methodology uses to carry up the experimental works is discussed in detail. The measurements of the signal performance are performed under different MS environments such as tree-shadowed and building-shadowed and hence the results are compared to the data obtained for open space environment. The analysis of the signal performance under different MS environments is performed with respect to the elevation and azimuth degree at different time under a clear sky condition. The analysis produced forms an important part in the studies of the signal performance. From this research work, we would like to propose a simple and low cost data acquisition system in order to encourage more experimental works to be carried out especially in the less developed countries.
\end{abstract}

Keywords-Mobile satellite; cost effective; tree-shadowed; buildingshadowed

\section{INTRODUCTION}

MS communication [1-3] operates in L-band signal. L-band refers to the frequencies between $1-2 \mathrm{GHz}$ and it is particularly effective in providing rapid and flexible communication through mobile and portable terminal or transportable earth station. Voice and data communication services can be provided through the link between the satellite and the ground terminal.

The advantage of the MSS over Fixed Satellite System (FSS) is that the coverage of the system is spotty over rural area. The connection exists while on the move and this reflects the reliability of the system to the mobile users [4]. Therefore, its reliability is higher and more flexible in term of navigation system on the moving vehicles.
Most of the experimental works have been carried out in the developed countries such as North America, Australia, Europe and Japan. However, very little data represents the less developed countries such as in Africa and Asia. In order to have a more accurate model to represent the MS environments, more experimental works are needed especially in the less developed countries.

Current methods used to carry out experimental works utilize the existing Geo-satationary satellite network or airborne platform such as airplane and helicopter. These methods are both complicated and expensive. Therefore, a simple and low-cost data acquisition system is proposed to be used to carry out measurement for different MS environment and also encourage more experimental works to be carried out in the less developed countries.

Building-shadowed happen when the signal propagation is blocked and absorbed by the building. Thus, only part of the signal will penetrate through the wall and thus will decrease MS signal-to-noise ratio (SNR). The signal blockage is increased with the decrease in the satellite elevation angle as shown in Figure 1. The wave propagation might be different for different parts of a building whereby there is a light-of-sight (LOS) in certain location outside the building while the other side is non-LOS (NLOS).

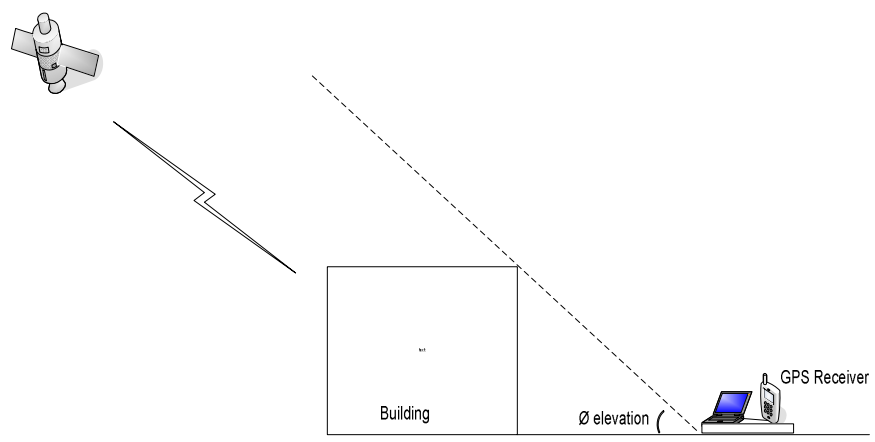

Figure 1. Building-shadowed propagation model based on the satellite elevation angle. 